\title{
Synaptogenèses et épigenèses cérébrales
}

> Aux mécanismes de plasticité cellulaire comJean-Pierre Bourgeois muns à toutes les cellules, les neurones ajoutent ceux de l'élaboration des formes et des fonctions qui leurs sont spécifiques. L'épigenèse synaptique est l'ensemble des ajustements morphofonctionnels des contacts synaptiques induits par l'environnement, dans la fenêtre de variabilité contrôlée par les réseaux de gènes, euxmêmes sélectionnés pendant l'évolution du cortex cérébral. Dans le paradigme dominant aujourd'hui, l'épigenèse synaptique constitue le mécanisme matériel du stockage des signaux représentant le monde environnant dans le cortex cérébral. La notion de périodes critiques au cours du développement ouvre l'inscription épigénétique de l'histoire de l'individu dans l'affinage final des formes et des fonctions de ses neurones. Cette «ouverture épigénétique», maximale dans le cerveau humain, est probablement la source de la très grande adaptabilité cognitive de notre espèce, mais peut-être aussi une de ses fragilités. «

Les neurones et leurs synapses se distinguent des autres cellules par trois caractéristiques, au moins: leurs positionnements dans le tissu nerveux, les configurations géométriques uniques de leurs arbres axonaux et dendritiques, et de leurs synapses (synapto-architectonie), et les distributions spatiales et temporelles des patrons de potentiels d'action (PPA) circulant dans ces arbres. Le développement et le maintien de ces trois paramètres assurent à l'individu ses apprentissages et sa permanence psychocognitive.

\section{Synapses «adultes » et épigenèse}

Le contact synaptique - la synapse - est le lieu morphologique d'apposition entre deux domaines subcellulaires de deux neurones distincts, caractérisé par des asymétries de structure et de fonction (Figure 1). C'est aussi un point d'articulation entre les contraintes

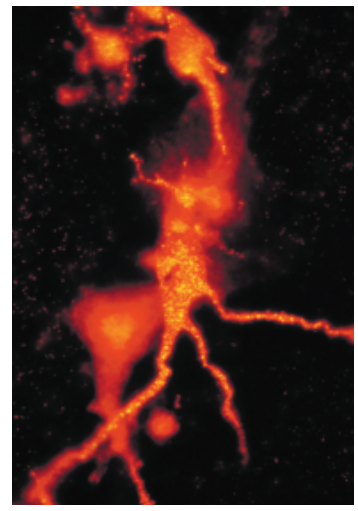

génétiques et métaboliques intracellulaires Laboratoire Récepteurs et cognition, Département des Neurosciences, Institut Pasteur, 25, rue du Docteur Roux, 75724 Paris Cedex 15. France. jpbourg@pasteur.fr et l'ensemble des contraintes liées au flux permanent et variable des PPA.

L'efficacité de la transmission synaptique varie épigénétiquement tout au long de la vie. Elle est modulée par la composition et le niveau de dépolarisation de la membrane plasmique postsynaptique. Elle est également modulée par la structure des PPA arrivant au niveau présynaptique et la probabilité $(p)$ de libération vésiculaire des neurotransmetteurs. La séquence temporelle et les niveaux d'activation de ces deux domaines pré- et postsynaptiques feront que l'efficacité de cette transmission synaptique sera augmentée, dans la potentiation synaptique à long terme (PLT), ou diminuée, dans la dépression synaptique à long terme (DLT). Ces variations, observées in vitro et in vivo, constituent depuis trente ans le paradigme dominant des bases morphofonctionnelles des apprentissages et des mémorisations dans le cerveau. Les débats théoriques et expérimentaux en cours portent sur la prévalence d'un domaine pré- ou postsynaptique sur l'autre, et sur la présence objective d'états fonctionnels discrets (c'est-à-dire distincts les uns des autres) de la synapse.

\section{Modèles d'états synaptiques discrets}

Dans ces modèles, les synapses peuvent basculer, réversiblement ou pas, dans divers états fonctionnels discrets. Toutes les transitions ne sont pas possibles, car certaines paraissent déterminées par la séquence temporelle des états précédents. De nombreux dia- 
grammes d'états ont été proposés ces dernières années [1-3]. Seuls quelques états et quelques mécanismes impliquant les récepteurs du $\mathrm{N}$-méthyl-D-aspartate (NMDA-R) et les récepteurs de l'acide $\alpha$ amino-3-hydroxy-5-méthyl-4-isoxazole proprionique (AMPA-R), dans les synapses excitatrices de l'hippocampe et du cortex cérébral des mammifères, seront évoqués ici (Figure 2).

Dans les synapses silencieuses «muettes» (Figure 2A), les NMDA-R et AMPA-R postsynaptiques peuvent être présents, mais la probabilité ( $p$ ) que le bouton présynaptique libère du glutamate vésiculaire est nulle $(p=0)$. La PLT enclencherait une augmentation de $p$, impliquant des signaux rétrogrades (c'est-à-dire circulant du domaine postsynaptique vers le présynaptique) comme le monoxyde d'azote (NO), I'ATP, et d'autres encore. La plupart des synapses silencieuses seraient «muettes» ou «murmurantes».

Dans les synapses silencieuses « sourdes» (Figure 2A), la libération vésiculaire de glutamate est suffisante $(p>0)$, mais il n'y a pas de dépolarisation postsynaptique au repos. Les AMPA-R sont absents de la membrane plasmique postsynaptique. Les NMDA-R sont présents mais, bloqués par le $\mathrm{Mg}^{2+}$, ne sont pas activables au potentiel membranaire de repos. Comme ces synapses n'ont pas d'activation à AMPA$R$, elles ne peuvent être «déprimées», ce qui les distingue des synapses «actives». En revanche, elles peuvent être «potentiées» comme les synapses «actives»: cette potentiation les fait basculer de l'état « silencieux » à l'état « récemment silencieux ».

Dans les synapses «récemment silencieuses» (Figure 2B), la PLT enclencherait un processus d'insertion et d'ancrage des AMPA- $R$ dans la membrane plasmique postsynaptique. Dans cet état, les AMPA-R deviennent activables en plus des NMDA-R dans la membrane postsynaptique. Ces synapses « récemment silencieuses » sont encore distinctes des synapses

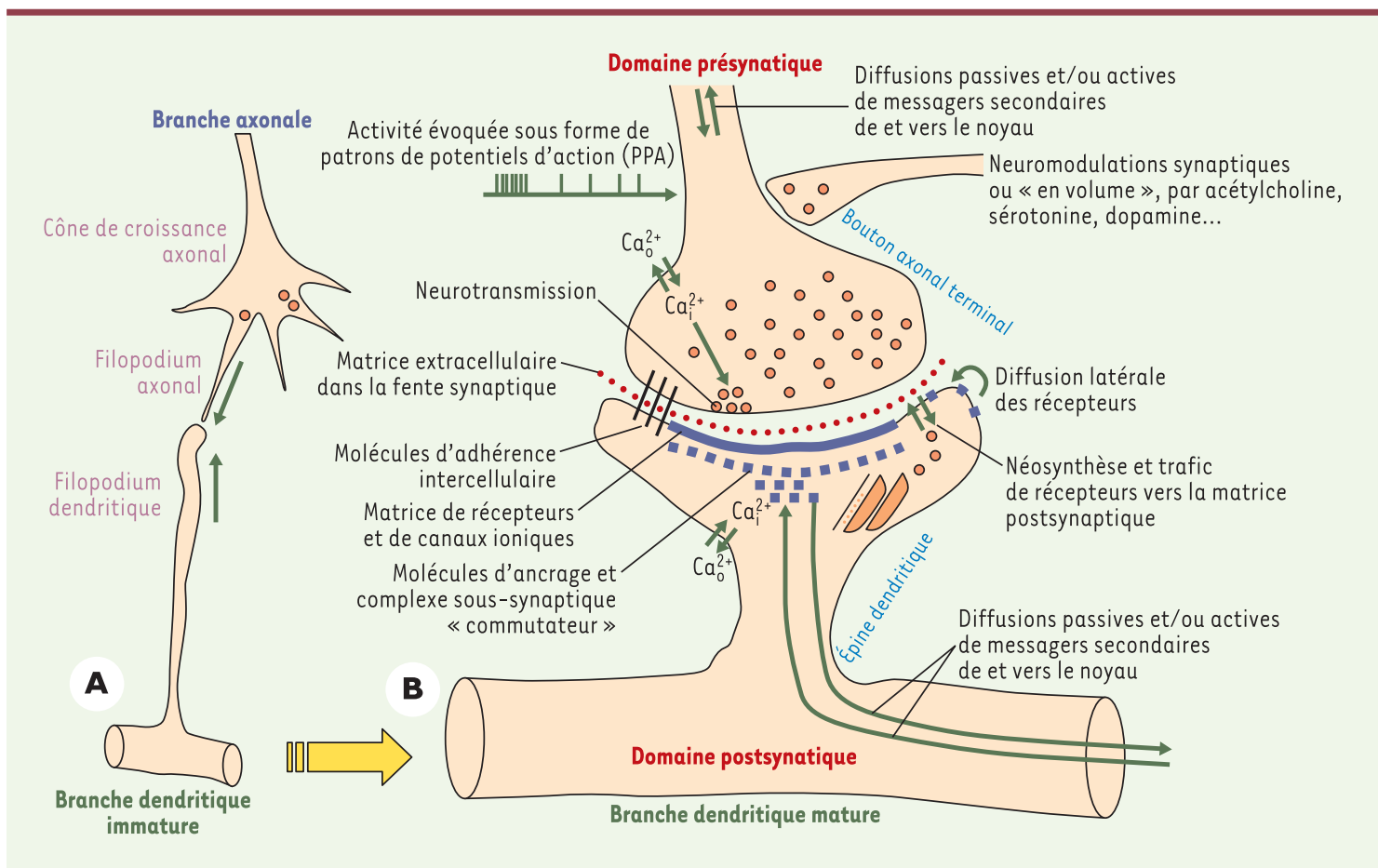

Figure 1. Représentations schématiques et partielles des synapses immatures et matures. A. La protosynapse est une des formes intermédiaires, immatures et labiles, de la synapse. Au début de la synaptogenèse, les filopodiums axonaux et dendritiques, très riches en cytosquelette d'actine, sont très mobiles et explorent, palpent leur environnement tissulaire jusqu'à la rencontre d'un partenaire neuronal cytocompatible. Lorsque cela arrive, la protosynapse se stabilise et s'agrandit en 45 à 60 minutes, par assemblage de nombreuses molécules d'adhérence intercellulaire, de récepteurs pharmacologiques ionotropiques et métabotropiques, de multiples canaux ioniques, et par échanges de facteurs trophiques diffusibles. B. La synapse mature est formée de deux domaines neuronaux pré- et postsynaptiques séparés par l'espace extracellulaire de la fente synaptique. Dans chaque domaine, de grands assemblages protéiques fonctionnent comme des commutateurs moléculaires, basculant la transmission synaptique vers divers niveaux d'efficacité [29]. Les mêmes mécanismes moléculaires sont à l'œuvre dans les synapses matures et immatures, mais, dans ces dernières, le cytosquelette en plein développement est beaucoup plus modulable, jusque dans les arbres axonaux et dendritiques. Les interactions entre les états fonctionnels des synapses et les niveaux d'activation des gènes se font par de multiples cascades de signaux importés de et exportés vers le génome (doubles flèches). 
« actives » en ce sens qu'elles ne peuvent pas basculer vers l'état « déprimé » par DLT. Elles basculent spontanément vers l'état « actif » en 30 minutes.

Dans les synapses «murmurantes», les NMDA-R et AMPA-R sont présents dans la membrane postsynaptique, mais sont peu activés car peu de glutamate est libéré au niveau présynaptique $(p \approx 0)$. La faible concentration de glutamate dans la fente synaptique peut activer les NMDA-R à forte affinité, mais pas les AMPA-R à faible affinité. La PLT entraînerait une ré-augmentation soit de la libération vésiculaire locale du glutamate $(p \neq 0)$, soit du déversement de glutamate provenant des synapses voisines.

Les synapses « actives » (Figure 2C) constituent l'état le plus plastique et pluripotent. Les NMDA-R et AMPA-R sont présents et actifs dans la membrane plasmique postsynaptique. La transmission présynaptique est très efficace ( $p$ > 0 ). Le mode d'activation des NMDA-R peut faire basculer rapidement ces synapses soit vers une potentiation (par PLT), soit vers une dépression (par DLT), selon les PPA circulants. Les NMDA-R sont impliqués dans le basculement vers l'état «déprimé», ce qui distingue les synapses «actives» des synapses «potentiées».

Dans les synapses «potentiées»(Figure 2D), les NMDA- $R$ et AMPA- $R$ sont présents et actifs dans la membrane plasmique postsynaptique. Le nombre d'AMPA-R y est significativement augmenté par diffusion latérale [4], trafic, insertion et ancrage de récepteurs préexistants ou nouvellement synthétisés dans l'épine dendritique: l'efficacité de la transmission synaptique est maximale. L'état «potentié» se distingue de l'état «actif» essentiellement par le fait que sa «dé-potentiation» et le basculement vers l'état «déprimé» s'effectue via des récepteurs glu- tamatergiques métabotropiques (mGluR). L'activation des mGluR conduit à une réduction du $\mathrm{Ca}^{2+}$ intracellulaire, entraînant une diminution de la libération vésiculaire présynaptique et une inactivation des NMDA-R postsynaptiques.

Les synapses «déprimées» (Figure 2E) peuvent re-basculer vers les états « actifs » et «potentiés » par PLT, comme cela a été décrit précédemment. Elles peuvent également basculer vers l'état «silencieux» par activation soutenue des mGluR. Des stimulations synaptiques prolongées dans le temps peuvent induire une synaptogenèse, avec des remaniements importants du cytosquelette d'actine, de nombreuses molécules d'ancrage, des AMPA-R... (Figure 2F). Chez l'adulte, cette synaptogenèse reste localisée aux seuls domaines pré- et postsynaptiques, sans remaniement global des arbres axonaux et dendritiques.

\section{Modèles d'états synaptiques en continuum}

D'autres modèles suggèrent qu'il n'y a pas de sauts discrets d'états fonctionnels, mais un continuum de niveaux de plasticité synaptique. Le flux continu de signaux à mémoriser ajuste continuellement et localement des modifications post-traductionnelles des protéines

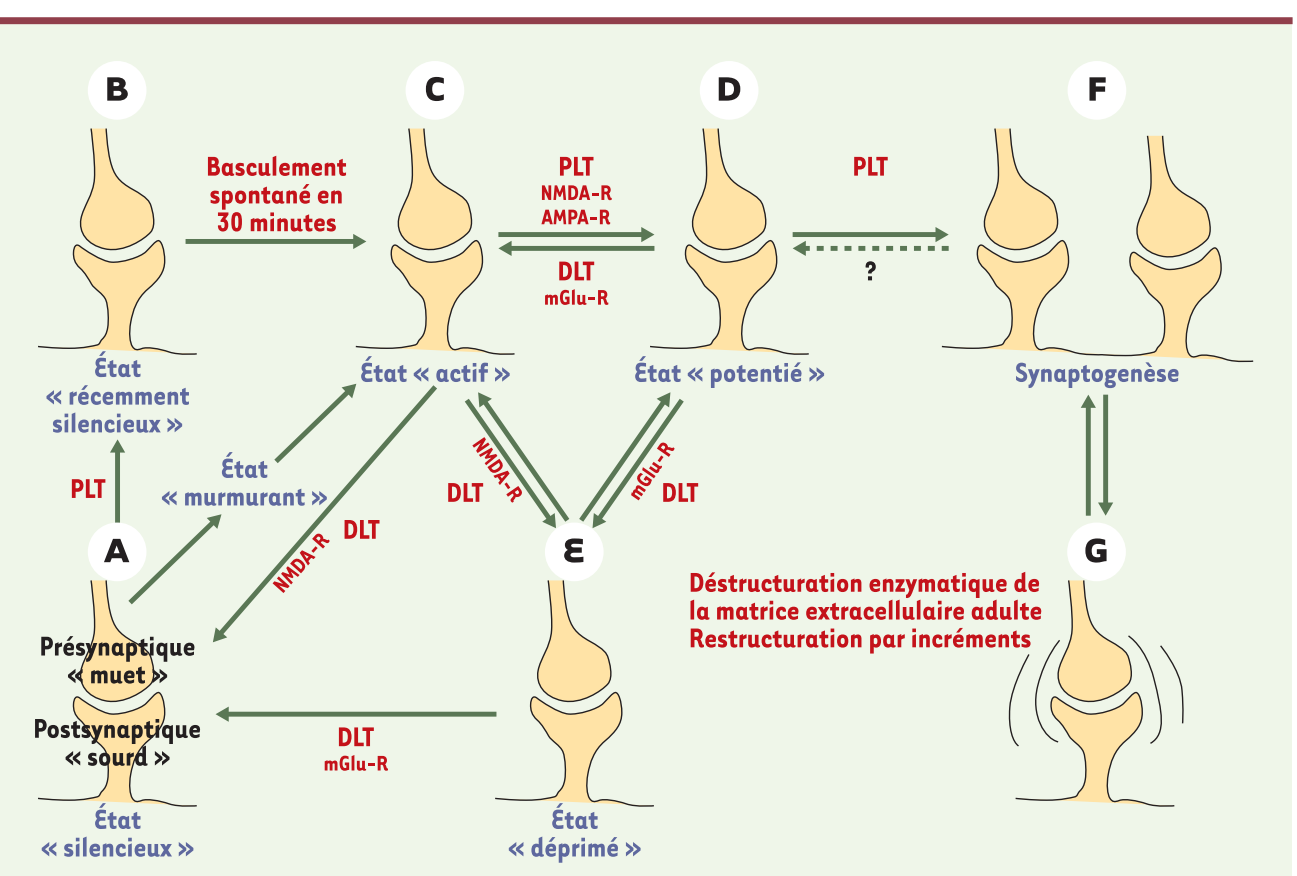

Figure 2. Diagramme des états fonctionnels possibles des synapses. Dans ces modèles de plasticité, les synapses basculent d'un état discret vers un autre selon le niveau et la séquence temporelle des activations locales des domaines pré- et/ou postsynaptiques $(A-\varepsilon)$ (voir texte). Pour la clarté du diagramme, tous les basculements possibles ne sont pas représentés. À cette plasticité locale s'ajoute une métaplasticité synaptique liée à l'état fonctionnel de chaque neurone, résultant de son histoire passée. Ces très nombreux états de plasticité accroissent considérablement la capacité «computationnelle» de chaque synapse. Ils permettent aux neurones d'ajuster leurs niveaux d'excitabilité instantanément (milliseconde) ou à long terme (années), localement (une synapse) ou globalement (assemblées de neurones), en fonction de l'activité évoquée circulant dans les circuits. Ils permettent d'inscrire, de maintenir et de restituer les traces mnésiques. Le débat sur la persistance et la distribution spatiale de ces états synaptiques, sorte de «phrénologie synaptique» [30], dans un flux constant de signaux, reste ouvert. Dans les cas de synaptogenèse observée chez l'adulte $(F)$, nous ne savons pas encore si la construction d'un nouveau réseau nécessite la destruction d'un autre (G) (diagramme d'après [1-5]). 
synaptiques [5]. Les mécanismes sont multiples: phosphorylation, palmitoylation, glycosylation, fucosylation, acétylation... Ces régulations par l'activité de chaque contact synaptique suffisent à inscrire et maintenir les traces mnésiques, les synthèses protéiques ne servant qu'à renouveler constamment les protéines synaptiques.

\section{Métaplasticité synaptique}

À la plasticité de chaque synapse s'ajoute une métaplasticité par coopérativité ou compétition avec les synapses voisines, pour le par-

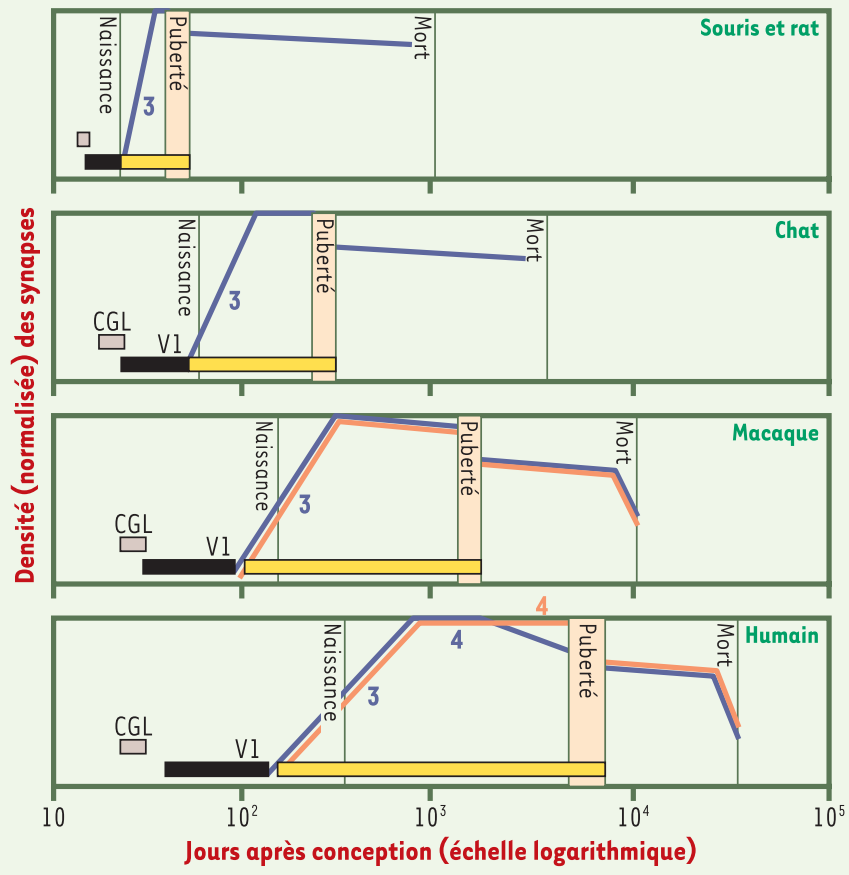

Figure 3. Synaptogenèse dans les cortex visuel primaire (traits bleus) et préfrontal (traits rouges) de cinq espèces de mammifères. La cinétique de la densité des contacts synaptiques (ordonnée cartésienne) est présentée en fonction du nombre de jours après la conception (abscisse logarithmique). Les rectangles noirs représentent les périodes de neurogenèse dans le cortex cérébral. Seules les phases 3 et 4 de la synaptogenèse sont désignées ici. Cette courbe-enveloppe simplifiée recouvre en fait de très nombreuses vagues successives de synaptogenèse distinctes pour divers réseaux neuronaux. Les phases fœtales les plus précoces sont indépendantes des interactions avec le monde extérieur. Les phases de la période prénatale jusqu'à la fin de la puberté comportent les périodes critiques (rectangles jaunes) de développement des réseaux neuronosynaptiques. Pendant ces périodes critiques, les interactions normales avec le milieu environnant et socioculturel sont nécessaires à la maturation correcte de ces circuits. Pendant la vie adulte, l'environnement peut toujours modifier les circuits synaptiques, mais seulement localement. Au cours de l'évolution, l'augmentation significative de la taille du cortex cérébral s'est accompagnée d'une augmentation importante de la durée de sa maturation. Les périodes critiques atteignent leurs plus longues durées, une vingtaine d'années, dans le cortex cérébral humain. CGL: corps genouillé latéral (thalamus visuel); V1: aire corticale visuelle primaire (aire 17 de Brodmann) (d'après [10-12]). tage de facteurs de maintenance diffusant dans le même dendrite et dans le milieu extracellulaire [6]. Divers neuromodulateurs (acétylcholine, sérotonine, dopamine...) peuvent également modifier les états fonctionnels des synapses en agissant différentiellement sur les compartiments pré- et postsynaptiques (Figure 1B). Par exemple, l'acétylcholine module de manière bidirectionnelle la PLT et la DLT [7] via des récepteurs nicotiniques et selon les proportions relatives des sous-unités $\alpha 7^{*}$ et $\alpha 4^{*}$, elles-mêmes contrôlées par l'activité [8]. Les interneurones inhibiteurs [9] et les cellules gliales participent aussi fortement à ces régulations épigénétiques. Ces très nombreux états de plasticité accroissent considérablement la capacité «computationnelle» de chaque synapse, c'est-à-dire la capacité d'augmenter, diminuer, filtrer, intégrer ou encore moduler les signaux moléculaires antérogrades et rétrogrades circulant entre ses deux domaines pré-et postsynaptiques.

\section{Synapses immatures et cinétique de la synaptogenèse}

Dans le cortex cérébral humain, les premières synapses sont observables très tôt, dès le $40^{\mathrm{e}}$ jour après la conception. La cinétique de synaptogenèse est complexe et comporte plusieurs phases distinctes, dont la plus rapide a lieu pendant la période périnatale (phase 3 dans la Figure 3 ). Le processus de production massive des synapses est très robuste [10-12], suggérant un contrôle génétique rigoureux [13], mais les gènes impliqués dans la synaptogenèse n'ont pas encore été identifiés. Beaucoup plus de synapses sont formées pendant le développement qu'il n'en restera chez l'adulte: en effet, de nombreuses vagues de synaptogenèse se succèdent [10-12] jusqu'à la puberté, où une perte significative de synapses a lieu (Figure 3). Les premières synapses formées sont majoritairement «silencieuses». Mais très tôt, dès le $7^{e}$ mois de gestation chez l'homme (phase 3 ), la croissance des réseaux neurono-synaptiques devient influençable par les PPA produits par tous les récepteurs sensoriels périphériques (représentations de l'environnement) et proprioceptifs (représentations du corps), ce que l'on nomme l'« activité évoquée ». Parmi les synapses en compétition les unes avec les autres pour des facteurs de maintenance, seules certaines seront stabilisées sélectivement [14]. L'épigenèse synaptique «ouvre» [15] alors les remodelages de la quantité et de la distribution tridimensionnelle des synapses (synaptoarchitectonie) aux apprentissages essentiels. 


\section{Périodes critiques}

La plasticité neuronale perdure tout au long de la vie, mais il existe des périodes critiques du développement pendant lesquelles la croissance et l'organisation des branches axonales et dendritiques deviennent extrêmement sensibles à la présence et à l'organisation normale des PPA y circulant. Les signaux représentant l'environnement du cerveau sont alors nécessaires pour permettre l'affinement des circuits synaptiques et leur fonctionnement optimal, et des altérations de l'environnement perturbent ou retardent leur maturation: par exemple, le développement dans un environnement de lumière stroboscopique, ou dans le noir, prolonge la période critique du développement du cortex visuel primaire [16]. Le développement dans un environnement sonore de «bruit blanc continu » prolonge celle du cortex auditif primaire $[17,18]$. Enfin, une déprivation maternelle ou paternelle à la naissance [19] modifie la distribution des épines dendritiques dans la couche II du cortex cingulaire chez le rongeur, et cette altération précoce persiste chez l'adulte. Pour chaque modalité sensorielle, motrice ou cognitive, existe une période critique spécifique et précise dans le temps.

Les mécanismes moléculaires contrôlant le début et la durée des périodes critiques sont multiples comme le montrent quelques exemples repris ci-dessous [20]. La libération d'un facteur de croissance, le BDNF (brain-derived neurotrophic factor), dans le cortex visuel de souris est liée à l'activité évoquée, et cette dépendance est amplifiée pendant la période critique de formation des colonnes de dominance oculaire ( $C D 0$, domaines du cortex visuel dominés anatomiquement et fonctionnellement par un œil ou l'autre). L'équilibre entre les flux de signaux antérogrades et rétrogrades importe plus que leurs quantités absolues: un excès de signaux trophiques rétrogrades peut entraîner une rétraction de branches axonales s'il n'est pas équilibré par un flux de signaux antérogrades ajusté. De son côté, le remplacement de la sous-unité NMDA- $R_{2 A}$ (courants courts) par la sous-unité NMDA- $R_{2 B}$ (courants longs) coïncide avec la fermeture des périodes critiques pour les déprivations sensorielles dans les aires corticales somato-sensorielles, auditives et visuelles. Au début du développement, l'inhibition des neurones par l'acide $\gamma$-amino butyrique (GABA) « ouvre » quant à elle la plasticité du système visuel. Par exemple, le cortex visuel des souris, dont le gène codant pour la L-glutamate décarboxylase (enzyme synthétisant le $\mathrm{GABA})\left(\mathrm{GAD} 65^{-/-}\right)$a été invalidé, est insensible aux déprivations monoculaires. La plasticité synaptique peut être restaurée par le diazépam, un agoniste GABAergique [21, 22]. Par ailleurs, l'expression de la protéine CPG15 (candidate plasticity gene 15) dans l'arbre axonal est contrôlée par l'activité évoquée circulant dans les voies visuelles: cette régulation est maximale pendant les périodes critiques de formation des colonnes de dominance oculaire dans le cortex visuel.

Les expressions des protéines du complexe majeur d'histocompatibilité de classe I ( $\mathrm{CMH}-\mathrm{I})$ et de leurs récepteurs porteurs de sous-unités $C D 3 z$, molécules d'adhérence et de signalisation intercellulaires, sont également activées par l'activité évoquée: cette sensibilité est maximale pendant les périodes critiques de développement des circuits neuronosynaptiques qui les expriment. On observe chez les souris $M H C-1^{-/-}$ou $\mathrm{CD}^{-1 /-}$ un défaut d'élagage des axones, une augmentation de la PLT et une absence de la DLT.

Les états fonctionnels des synapses sont reliés aux régulations de réseaux de gènes par de multiples cascades de signaux intracellulaires transportés vers le noyau (Figure lB). Par exemple, une déprivation monoculaire entraîne une activation de l'expression de gènes sous le contrôle de CRE ( $C A M P$ regulated elements); ce contrôle de la transcription par les facteurs de type CREB existe seulement pendant la période critique de formation des CDO dans le cortex visuel [20].

Les périodes critiques - de quelques semaines chez la souris (Figure 3) - atteignent leur durée maximale, une vingtaine d'années, dans le cortex cérébral humain [10-12].

\section{Pouvoir réouvrir des périodes critiques dans le cerveau adulte?}

Les neurobiologistes apprennent à maîtriser expérimentalement l'ouverture et la fermeture des périodes critiques pour réinstaurer une épigenèse synaptique dans le cortex cérébral adulte $[21,22]$.

L'ouverture des périodes critiques peut être avancée ou prolongée par des traitements pharmacologiques utilisant des agonistes GABAergiques tels que le diazépam. La dépolymérisation de la matrice extracellulaire par traitement enzymatique, associée à un apport de facteurs de croissance, permet aux réseaux neurono-synaptiques de recouvrer une certaine plasticité (Figure 2F et 2G) [23].

Un environnement enrichi en odeurs peut stimuler la neurogenèse et la synaptogenèse dans le système olfactif adulte [24]. La restauration de la plasticité synaptique dans une modalité sensorielle donnée, par exemple dans le cortex visuel, peut également être stimulée par l'activation d'autres modalités sensorielles, motrices, et cognitives, dans un environnement enrichi $[25,26]$.

La restauration peut s'effectuer en privilégiant le paramètre temps: des réapprentissages par incréments [27], sur une longue durée, peuvent réorganiser les circuits neurono-synaptiques. Les thérapies comportementales cognitives peuvent aussi réorganiser, au moins fonctionnellement, des circuits corticaux [28]. 


\section{Conclusions}

La synapse est beaucoup plus qu'une simple solution de continuité anatomique et physiologique locale entre deux neurones. L'épigenèse synaptique permet d'intégrer sur un même neurone les flux permanents des signaux des diverses modalités sensorielles, motrices, végétatives, cognitives... représentant à chaque instant le monde environnant, et de les mêler constamment aux représentations durables de l'histoire individuelle. Ces associations servent aussi les fonctions cognitives les plus abstraites, permettent des combinaisons nouvelles de représentations et constituent les bases matérielles des capacités créatrices du cerveau humain. La façon dont l'épigenèse synaptique code anatomiquement et fonctionnellement ces représentations est encore inconnue. Les manipulations épigénétiques sur le cerveau adulte ouvrent des perspectives thérapeutiques fascinantes et constituent l'un des grands chantiers de la neurobiologie du XxI ${ }^{e}$ siècle. $\diamond$

\section{SUMMARY}

\section{Brain synaptogenesis and epigenesis}

Synaptic plasticity, or epigenesis, is present and varies throughout the whole life of the cerebral cortex. The adult synapse is formed of large and variable proteins assemblies acting as molecular switches leading to many distinct functional states. In the flow of activity circulating through the synaptic circuits, these multiple synaptic states transitions are modulated by the levels and sequences of activations of the preand post-synaptic domains. The efficiency of synaptic transmission is also modulated by competition and/or cooperativity with neighbouring synapses, and by many neuromodulations. Some transitions eventually lead to synaptogenesis. In the adult cerebral cortex, synaptogenesis remains a local event; axonal and dendritic arbors are not reshaped. On the contrary, during pre- and post-natal synaptogenesis, the same molecular mechanisms lead to a significant reorganization of the axonal and dendritic arbors. Early in the development, synapses are generated and differentiate under the control of robust mechanisms governed by genes. Then, during the critical periods, extending from the end of gestation to the end of puberty, the refinment of the synaptic architecture becomes experience-expectent. This «epigenetic opening» of synaptogenesis to environment is maximal in the human brain. It is the source of the exceptional cognitive adaptability of our species, and possibly one of its major fragility. Epigenetic manipulations of these critical periods are undertaken, allowing restoration of synaptic plasticity also in the adult brain. $\diamond$

\section{RÉFÉRENCES}

1. Lisman J. Long-term potentiation: outstanding questions and attempted synthesis. Phil Trans R Soc Lond B 2003; 358: 829-42.

2. Montgomery JM, Madison DV. Discrete synaptic states define a major mechanism of synapse plasticity. Trends Neurosci 2004; 27 : 744-50.

3. Voronin LL, Cherubini $\varepsilon$. «Deaf, mute and whispering 》 silent synapses: their role in synaptic plasticity. J Physiol 2004; 557: 3-12.

4. Groc L, Heine M, Cognet L, et al. Differential activity-dependent regulation of the lateral mobilities of AMPA and NMDA receptors. Nat Neurosci 2004; 7: 695-6.

5. Routtenberg A, Rekart JL. Post-translational protein modification as the substrate for long-lasting memory. Trends Neurosci $2005 ; 28: 12-9$.

6. Turrigiano G. A competitive game of synaptic tag. Neuron 2004 ; 44: 903-4.

7. Salgado-Commissariat D, Rosenfield DB, Helekar SA. Nicotine-mediated plasticity in robust nucleus of the archistriatum of the adult zebra finch. Brain Res 2004; 1018: $97-105$

8. Sallette J, Pons S, Devillers-Thiery A, et al. Nicotine enhances intracellular nicotinic receptor maturation: a novel mechanism of neural plasticity. Neuron 2005 (sous presse).

9. Hensch TK, Stryker MP. Columnar architecture sculpted by GABA circuits in developing cat visual cortex. Science 2004; 303: 1678-81.

10. Bourgeois JP. Synaptogenesis, heterochrony, and epigenesis in the mammalian neocortex. Acta Paediatr 1997; 422 (suppl): 27-33.

11. Bourgeois JP. Synaptogenesis in the neocortex of the newborn. In: Nelson CA, Luciana M, eds. Handbook of developmental cognitive neuroscience. A Bradford book. Cambridge, Massachusetts: MIT Press, 2001: 23-34.

12. Bourgeois JP. Le développement de la connectivité cérébrale: étape ultime de I'individuation? In: Changeux JP, ed. Gènes et culture. Paris: Éditions Odile Jacob, 2003: 93-115.

13. Goldberg JL. Intrinsic neuronal regulation of axon and dendrite growth. Curr Opin Neurobiol 2004; 14: 551-7.

14. Changeux JP, Danchin A. Selective stabilization of developing synapses as a mechanism for the specification of neuronal networks. Nature 1976; 264: 705-12.

15. Changeux JP. L'homme de vérité. Paris: Éditions Odile Jacob, 2002.

16. Fagiolini M, Pizzorusso T, Berardi N, et al. Functional postnatal development of the rat primary visual cortex and the role of visual experience: dark rearing and monocular deprivation. Vision Res 1994; 34: 709-20.

17. Chang $\varepsilon F$, Merzenich MM. Environmental noise retards auditory cortical development. Science 2003; 300: 498-502.

18. Wang $X$. The unexpected consequences of a noisy environment. Trends Neurosci $2004 ; 27: 364-6$.

19. Helmeke C, Ovtscharoff W Jr, Poeggel G, Braun K. Juvenile emotional experience alters synaptic inputs on pyramidal neurons in the anterior cingulate cortex. Cerebr Cortex $2001 ; 11: 717-7$.

20. Berardi N, Pizzorusso T, Maffei L. Critical periods during sensory development. Curr Opin Neurobiol 2000; 10: 138-45.

21. Hensch TK. Controling the critical period. Neurosci Res 2003; 47: 17-22.

22. Hensch TK. Critical period regulation. Ann Rev Neurosci 2004; 27 : 549-79.

23. Tropen D, Caleo M, Maffei L. Synergistic effects of brain-derived neurotropic factor and chondroitinase $A B C$ on retinal fiber sprouting after denervation of the superior colliculus in adult rats. J Neurosci 2003; 23: 7034-44.

24. Rochefort C, Gheusi G, Vincent JD, Lledo PM. Enriched odor exposure increases the number of newborn neurons in the adult olfactory bulb and improves odor memory. J Neurosci 2002; 22: 2679-89.

25. Cotman CW, Berchtold NC. Exercise: a behavioral intervention to enhance brain health and plasticity. Trends Neurosci 2002; 25: 295-301.

26. Bartoletti A, Medini P, Berardi N, Maffei L. Environmental enrichment prevents effects of dark-rearing in the rat visual cortex. Nat Neurosci 2004; 7: 215-6.

27. Linkenhoker BA, Knudsen $\varepsilon$. Incremental training increases the plasticity of the auditory space map in adult barn owls. Nature 2002; 419: 293-6.

28. Paquette V, Lévesque J, Mensour B, et al. Change the mind and you change the brain: effects of cognitive-behavioral therapy on the neural correlates of spider phobia. Neuroimage 2003; 18: 401-9.

29. Grant SGN, O'Dell TJ. Multiprotein complex signaling and the plasticity problem. Curr Opin Neurobiol 2001; 11:363-8.

30. Abraham WC, Robins A. Memory retention: the synaptic stability versus plasticity dilemna. Trends Neurosci 2005; 28: 73-8.

TIRÉS À PART

J.P. Bourgeois 